\title{
ABSOLUTELY FREE EXTRINSIC EVOLUTION OF PASSIVE LOW-PASS FILTER
}

\author{
Yerbol Sapargaliyev \\ School of Engineering and Design \\ Brunel University \\ Uxbridge, Middlesex, UB8 3PH \\ email: yerbol.sapar@brunel.ac.uk
}

\author{
Tatiana Kalganova \\ School of Engineering and Design \\ Brunel University \\ Uxbridge, Middlesex, UB8 3PH \\ email: tatiana.kalganova@ brunel.ac.uk
}

\begin{abstract}
Evolutionary electronics is a brunch of Evolvable Hardware, where the Evolutionary Algorithm is applied towards electronic circuits. The success of evolutionary search most of all depends on variable length representation methodology. The low-pass filter is a standard task in Evolutionary Electronics to start with. The results of evolution enable one to qualify whether the methodology is good for further experiments.

In this paper the maximum freedom for evolutionary search has been proclaimed as a main target during development of new VLR methodology. The introduction of $R$ support elements enables to perform an unconstrained evolution of analogue circuits for the first time. The proposed algorithm has been tested on the example of analogue low-pass filter.

The experimental results demonstrate that the evolved filter is comparable with filters evolved previously using Genetic Programming and Genetic Algorithms techniques. The obtained results are compared in details with low-pass filters previously designed.
\end{abstract}

Keywords: Evolvable hardware; evolutionary electronics; unconstrained evolution; analogue passive low-pass filter.

\section{Introduction}

Evolvable Hardware (EHW) is one of the most promising areas of today's electronics. Evolutionary Algorithm (EA) applied towards reconfigurable hardware enables to find a solution among global solution space. The EHW where the ultimate goal is a circuit design is also referred as Evolutionary Electronics [3]. The evolutionary electronics gives an alluring opportunity for an amateur in the field of Electronics to reach out the same results as professional one, possessing mostly the knowledge of Darwinian's laws and inspiration. The EA, navigated by a fitness values, provides randomly created and mutated chromosomes. Each chromosome encodes the structure for a circuit and has to be evaluated by a fitness function assigning a fitness value. The last one shows how close the current hardware structure by its behavioral and circuit characteristics to the required one. The circuits evolved may have unconventional designs and less of all depend on personal knowledge of a designer [1] - [6].

For instance, using simulation software (extrinsic EHW), low-pass filters [1]-[6] and amplifiers [2]-[4] are successfully designed with the help of EA. In [8] the unconstrained evolution, both spatially and temporally, has been applied intrinsically towards the digital FPGA-based reconfigurable hardware. By releasing the full repertoire of behaviors that FPGA can manifest, namely, allowing any connections among modules, letting the evolution to evolve the granularity of modules as well as the regimes of synchronization, evolution has been able to find a highly efficient electronic structure, which requires 1-2 orders less silicon area to achieve the same performance as conventional design does.

In analogy to this approach, the unconstrained evolution could be applied towards the originally analogue circuits. In this sense, the range of circuit-structure-checking rules at the netlist composition stage, prohibiting the invalid circuit graphs, are regarded as the main constraints for the design methodology. In this paper, we consider unconstrained extrinsic evolution for analogue "LCR" circuit design of lowpass filter. We utilize the simplest increasing length genotype (ILG) sweeping strategy [2] that together with unconstrained evolution produces promising results in comparison with previously achieved ones.

The paper is organized as follows. The next section gives an overview of previous work in the area. Section 3 introduces the unconstrained evolutionary technique. Section 4 describes the experimental results and compares them with the filters evolved previously as well as designed conventionally. And, finally, the last section concludes the paper.

\section{Previous work on analogue filters evolution}

As well as in any evolutionary search, in electronic circuit design the freedom of evolutionary search is respected as crucially important for successful results. In [1], [2], [4] the freedom of search is emphasized, but has not been realized completely.

The considerable results were obtained by Koza et al in [1]. They used Genetic Programming (GP) circuit-constructing program trees with four kinds of circuit-constructing functions and automatically defined functions. Last one let them to evolve the filters with regular structures within the circuit. They utilized a procedure providing the DC path to ground from each node of a circuit by adding the giga-Om resistance, allowing any kind of connections among capacitors. This lets to avoid the most of "node floating" errors and reach the 
amount of invalid circuit graphs up to $2 \%$. Later, they simplify each circuit by removing redundant resistances and replacing all series and parallel compositions. The main drawback of this experience is that the technique requires large computing power to adopt the same methodology as well as the methodology by itself is very complex for implementation.

The larger computational efforts in a circuit evolution required by GP were proven by works of Zebulum et al [3] and Ando et al [5], where they have given the comparison between GP and Genetic Algorithm (GA). The first work was made as analogy to biology concept with comparison of performance among different types of sweeping strategies: Increasing Length Genotypes, Oscillating Length Genotypes and Uniformly Distributed Initial Population. The second work there was intrinsic evolution of real hardware for robustness purposes.

The analysis of previous development in evolution of analogue circuits design reveals that all the approaches developed in analogue circuit domain previously are based on the circuit-structure-checking rules for avoiding the invalid circuit graphs. In contrast, the experimental results were promising for evolution of digital circuits in the unconstrained search space [8]. Furthermore, the approaches introduced previously for analogue circuit design provided search with GP and GA, and none of them used purely ES.

Most of the developments in the analogue circuit design start from evolving a passive low-pass filters, that is a convenient tool for probation of evolutionary technique and tuning the EA parameters towards the more sophisticated designs [1], [2]. The behavior of low-pass filters between frequencies $1 \mathrm{~Hz}$ and $100 \mathrm{KHz}$, cut-off frequency $1 \mathrm{KHz}$ and transition band $1 \mathrm{KHz}$ has been actively researched through in [1]-[3]. Thus, the performance of proposed evolutionary technique could be evaluated more precise if the evolution target will have exactly the same filter properties.

\section{Unconstrained evolution of "LCR" circuits}

\subsection{Chromosome representation}

The basic idea of proposed algorithm is initially to generate the so called "embryo-circuits" and randomly grow them up by adding one-by-one elements. The embryo-circuit refers to the elements that are definitely known as essential for the target circuit, stay unchangeable during all the evolution and take place in each circuit netlist. In our case of LCR low pass filter as the target circuit, there are 3 such kind of elements: $\mathrm{AC}$ voltage source, source resistance and load resistance. We define the embryo circuit similar to the most popular case [1], [2], [4]-[6], where the circuit is driven by an incoming AC voltage source with a $2 \mathrm{~V}$ amplitude, has the source resistance $\mathrm{R}_{\text {source }}=1 \mathrm{k} \Omega$ and the load resistance $\mathrm{R}_{\text {load }}=1 \mathrm{k} \Omega$. The output voltage is measured on the pins of $R_{\text {load }}$.

The linear circuit representation is proposed for use, similar to one that exploited in [3]. Every element of a circuit is represented as a particular gene, and each of 4 element's features: name of an element, its parameter, and every its pin is represented by particular loci in a gene. The advantage of this chromosome representation is that it directly maps the description of one element in the Pspice netlist. The number of genes in chromosome equals to the number of elements in a circuit. The circuit depicted on Fig. 1 contains four elements (apart from embryo-elements). Therefore the chromosome representation contains four genes. Each gene is described by four loci. For instance, the first gene is " $\mathrm{L}-0 \quad 1 \quad 2 \quad 1.8 \mathrm{e}-1$ ", which describes the first inductor with pins $\mathrm{N} 1=1$ and $\mathrm{N} 2=2$ and with a value of $1.8 \mathrm{e}-1$ Henry. The first loci is reserved for the element's type ( $\mathrm{L}$ or $\mathrm{C}$ or $\mathrm{R}$ ), the second and the third loci are reserved for the first and the second pins, and the last loci is for the element's parameter. Similarly the remaining genes in the chromosome are encoded.

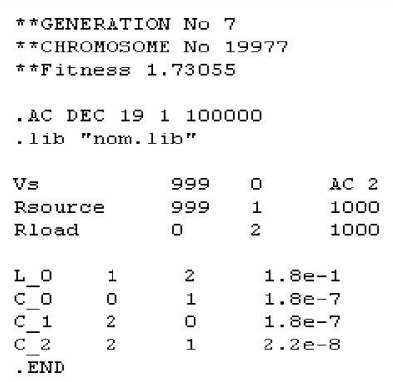

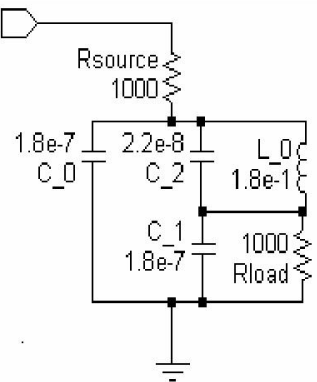

Figure 1. Chromosome representation at constrained evolution. The typical netlist (a) and the correspondent schematic (b). The three first elements $\left(V_{s}, R_{\text {source }}\right.$ and $\left.R_{\text {load }}\right)$ compose the emryocircuit.

\subsection{The essence of unconstrained evolution in "LCR" circuit design domain}

We call absolutely unconstrained evolution of an analogue circuit the process of a circuit netlist generation during which no any circuit-structure-checking rules applied and all the circuits are counted as valid graphs except ones that have elements with dangling nodes and with isolated subcircuits. There are two main kinds of invalidities that unacceptable for most of simulation software: the nodes that have no DC path to ground (tackled in [1]) and loops that involve inductors and/or voltage source. The most of methodologies in the area just prohibit such kinds of invalidities to appear. In the case of "LCR" circuits, the adding to each capacitor the Giga-Ohm resistance in parallel and the adding to each inductor the Micro-Ohm resistance in series, at the stage of Pspice cir-file generation, allows to avoid these invalidities. We call such kind of resistance as R-support. Using R-support and avoiding the dangling nodes makes almost any randomly generated circuit as valid, and indeed becomes an absolutely unconstrained. Fig.2 demonstrates how unconstrained evolution generates the circuits with R-support. The circuit depicted on Fig.1 once being processed by unconstrained evolution will have the view shown on Fig.2. Each element line describing inductor ( $\mathrm{L}_{-} 0$ ) is followed by R-support element (Rl_1) in series with infinitesimal parameter; and each 
element line describing conductor $\left(\mathrm{C}_{-} \mathrm{No}\right)$ is followed by $\mathrm{R}$ support element (Rc_No) in parallel with infinite parameter.

If the circuit-structure-checking rules are applied to a circuit after fitness assignment as a part of the simplification procedure, that is, every R-support element is checked whether it could be removed without damage to the current fitness value of a circuit, then the circuit could contain a few Rsupport elements or even does not contain them at all. For instance, the circuit shown on the Fig.2 becomes the circuit depicted on the Fig. 1 after such the simplification process.

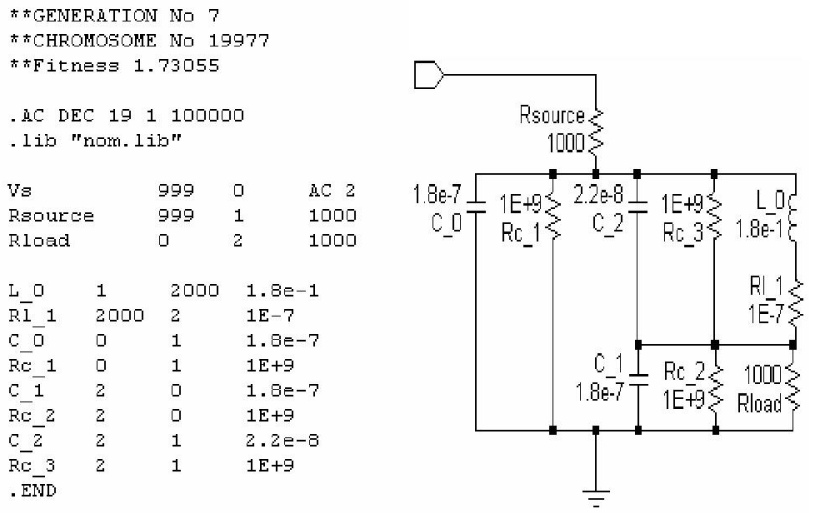

Figure 2. Chromosome representation at unconstrained evolution. The typical netlist and the correspondent schematic with R-support elements.

\subsection{Mutation}

The whole process for both constrained and unconstrained evolutions consists of two types of operations over the circuit to inculcate the simplest ILG sweeping. Every time the best fitness of a generation is not improved, the population falls into "Add new element mutation" (ANEM) procedure, i.e. one randomly generated gene is added to each chromosome except the chromosome with the best fitness value.

The "circuit structure mutation" (CSM) performs mutation over any of four loci of randomly chosen gene. If the mutation comes to a pin connection, the whole structure of a circuit is changed. However the total amount of elements stays unchangeable, the number of nodes of a circuit could be reduced or increased.

At the start the population comes to ANEM for preliminary circuit growth, and, after evaluation, it falls into CSM. The following switching rule between ANEM and CSM does manage: whenever the mutation within CSM does not bring any improvement in the fitness, the algorithm immediately switches to ANEM. Experimental results show that a fitness of an individual never improves by infinitesimal values, what makes the switch period between two kinds of mutations finite in time. While CSM searches for the best circuit within the given amount of elements, it unavoidable brings to a fitness improvement stuck. But the adding one element to a circuit during ANEM stage significantly improves the best fitness value reviving the whole process.

\subsection{Fitness Function}

We perform the AC-analysis along 96 points between $1 \mathrm{~Hz}$ and $100 \mathrm{kHz}$ (19 per decade), and measure the absolute deviation voltage between ideal value and produced by Pspice. We set the fitness evaluation in the analogy with [1], i.e. we distinguish as acceptable a voltage in the passband between $970 \mathrm{mV}$ and $1 \mathrm{~V}$ and a voltage in the stop-band between $0 \mathrm{~V}$ and $1 \mathrm{mV}$ :

$$
F_{1}=\sum_{i=0}^{p}\left|V_{\text {ideal }}^{i}-V_{\text {measured }}^{i}\right|,
$$

where $V_{\text {ideal }}^{i}$ is the voltage in $i$-th point in ideal filter and $V_{\text {measured }}^{i}$ is the voltage in the $i$-th point obtained for evolved filter; $p$ is a number of points evaluated in both stop and pass bands and equal to 96 . And we regard voltage at any other location as unacceptable, punishing it as follows:

$$
F_{2}=10 \times F_{1} \text {. }
$$

The transition band, consisting of five points between $1 \mathrm{kHz}$ and $2 \mathrm{kHz}$, is regarded as the "don't care" band, where the fitness is supposed to be equal zero.

\section{Experimental results}

The purpose of this experiment is to apply the new methodology to evolve a low-pass filter described in Section 3. Population size of 20,000 chromosomes is set in all experiments. Experimentally it has been established that the disruptive selection scheme [7] is the best: only $9 \%$ of the best chromosomes and $1 \%$ worst ones are to be chosen for the next generation. Being chosen for the next generation each chromosome contributes $10 \%$ of the next population size. Static mutation rate of $5 \%$ then applied to each chromosome.

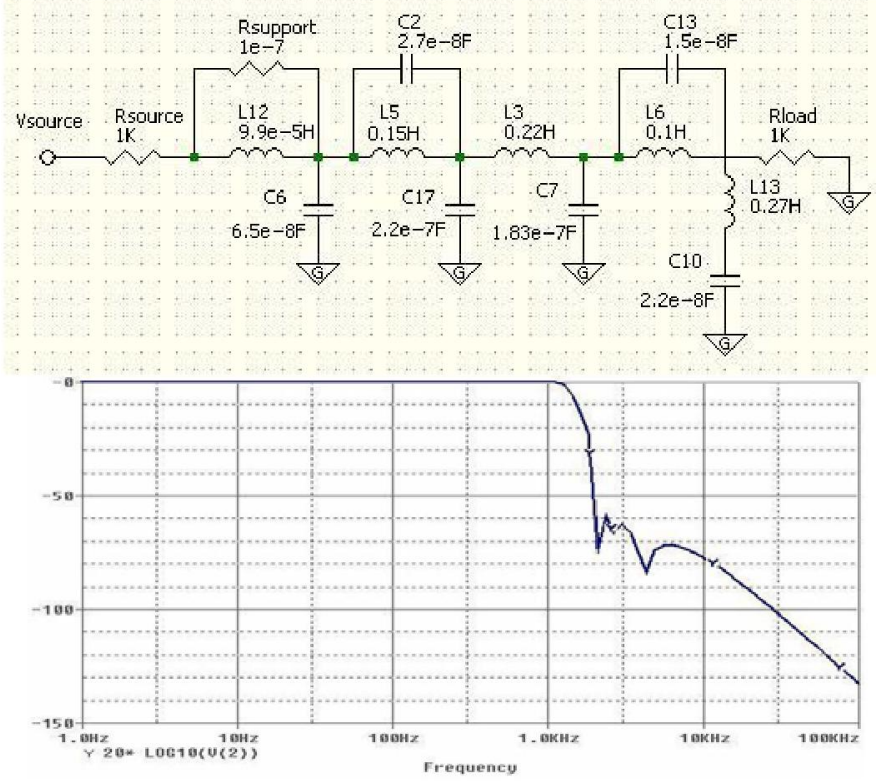

Figure 3. The schematic after simplification and the voltage response of the best low-pass filter evolved. 
Table 1. Comparison table of filter and evolution characteristics among works published before and present.

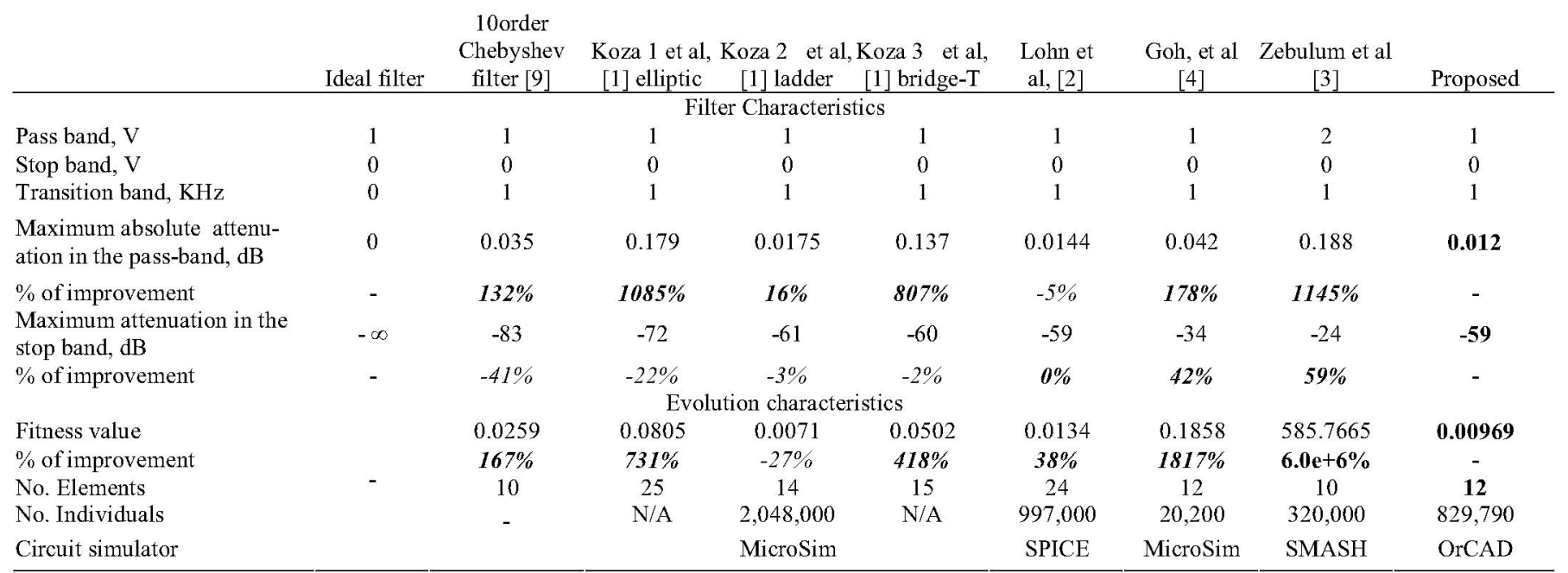

The value "\% of improvement" shows the correlation of difference between the value above in the same column and the correspondent value in the column "Proposed".

The best result out of 5 attempts has been obtained at chromosome 9,790 of generations 42 $(20,000 \times 41+9,790=829,790$ individuals $)$, with 12 elements before simplification (without embryo), among which one is Rsupport, and with fitness value 0.009693 . The schematic after simplification and the voltage response of the best circuit are shown on Fig.3. The non-monotonic filter has the following features: the maximum absolute attenuation in passband is $0.012 \mathrm{~dB}$, the maximum attenuation in stopband is $-59 \mathrm{~dB}$.

During the experiment, the amount of invalid graphs among all randomly generated ones did not exceed in average $0.03 \%$.

In order to provide fair comparison between obtained and previously published results, we validate each result using OrCAD Pspice-10.3. By this we have got filter characteristics for each circuit and its fitness values, all summarized in Table 1. The correct performance of the fitness function is verified by perfect match between the fitness value we have got and the fitness value published in [1] for ladder low-pass filter.

The achieved filter by its parameters is close to Lohn's one [2], but methodology complexity is in order simpler, the number of evaluations required to obtain the solution is $20 \%$ lower and the element amount is twice lower.

\section{Conclusions}

The process of extrinsic evolutionary design of analogue circuits before always been constrained to generation only the valid circuit graphs. However the introduction of R-support elements can significantly release these constraints.

The proposed technique based on Evolutionary Strategy in combination with increasing length genotype sweeping strategy produced the promising results. Thus, the instinctive wish to reduce the potential solution space for evolutionary search, by which usually the circuit-structure-checking rules are justified, is not always the best strategic maneuver for reducing the search time and obtaining good results.

\section{References}

[1] J. R Koza, F.H. Bennett III, D. Andre, M.A. Keane, and F. Dunlap, "Automated Synthesis of Analog Electrical Circuit by Means of Genetic Programming," IEEE Trans. Evolutionary Computation, Vol.1, No.2, 1997.

[2] J. D. Lohn, S.P.Colombano, "Automated Analog Circuit Synthesis using a Linear Representation," Proceedings of the 2nd Int'l Conference on Evolvable Systems: From Biology to Hardware, Springer-Verlag, Berlin, 1998, pp.125- 133.

[3] R. S. Zebulum, M.A. Pacheco, M. Vellasco, "Comparison of different evolutionary methodologies applied to electronic filter design," IEEE Trans. on Conference on Evolutionary Computation, Piscataway, NJ: IEEE Press, 1998, pp. 434-439.

[4] C. Goh, Y. Li, "GA automated design and synthesis of analog circuits with practical constraints," Proceedings of the 2001 Congress on Evolutionary Computation, Vol. 1, pp. 170-177, 2001.

[5] S. Ando, H. Iba, "Analog Circuit Design with a Variable Length Chromosome," Congress on Evolutionary Computation, pp.994-100, IEEE Press, 2000.

[6] J. B. Grimbleby, "Hybrid genetic algorithms for analogue network synthesis," Congress on Evolutionary Computing, Washington USA, pp. 1781-1787, 1999.

[7] T. Kuo, S.-H. Hwang, "Using disruptive selection to maintain diversity in genetic algorithms," Appl. Intel. 7, 1997, pp. 257-267.

[8] A. Thompson, ARTIFICIAL EVOLUTION IN THE PHYSICAL WORLD. In Gomi (Ed.): Evolutionary Robotics, AAI Books, 1997.

[9] LC filter design. Available at:

http://www-users.cs.york.ac.uk/ fisher/lcfilter/ 